\title{
Authoring of interventions with on-demand screen generation for mobile devices
}

\author{
Bruna C. R. Cunha ${ }^{1,2}$, Kamila R. H. Rodrigues ${ }^{1}$, Leonardo F. Scalco ${ }^{1}$, Isabela Zaine ${ }^{1}$, Caio C. Viel ${ }^{1}$, \\ Maria da Graça C. Pimentel ${ }^{1}$ \\ Universidade de São Paulo ${ }^{1}$, São Carlos, Brazil \\ Instituto Federal de São Paulo², São Paulo, Brasil \\ \{brunaru7,kamila.rios,leoscalcoxpe,isabela.zaine,caioviel\}@gmail.com,mgp@usp.br
}

\begin{abstract}
In this paper we present the ESPIM system, a set of tools that enables the authoring of intervention programs with focus on health and education. Created programs are played in a mobile app that generates screens on demand, according to their definition provided by JSON files. Using state-of-art technology we aim to provide a platform that can be used by professionals of health and education with no programming skills. To achieve that the system presents an intuitive authoring interface, built with requirements obtained with experts in different areas and with continuous feedback from evaluations, and a friendly mobile app, which presents elements that users are familiar and a good aesthetic design to motivate them. The system is being successfully used by professionals in health-related areas and shows many advantages in comparison to similar platforms.
\end{abstract}

\section{KEYWORDS}

ESM, mobile interventions, authoring, automatic screen generation

\section{INTRODUÇÃO}

A crescente democratização do uso de smartphones tornou a área de mhealth, a qual utiliza dispositivos móveis para ofertar serviços de saúde, uma área de pesquisa fortemente presente na realidade das pessoas, promovendo a diminuição da distância entre pesquisadores, profissionais de saúde e população. O sucesso e popularização da área é constatado pela grande disponibilidade de aplicativos relacionados nas lojas dos sistemas Android e iOS, assim como a grande quantidade de artigos científicos [12] publicados nos últimos anos.

Pesquisas envolvendo aplicativos de saúde para dispositivos móveis demonstram que o uso de intervenções pode aumentar a motivação de indivíduos [10], além disso, o uso de dispositivos móveis para promover intervenções é capaz de diminuir disparidades de saúde entre grupos mais vulneráveis [13].

No contexto da saúde, a coleta de informações e o provimento de informações personalizadas para indivíduos com demandas específicas permite aprimorar o tratamento de pacientes. Porém, a utilização de papel para este fim é muitas vezes pouco eficiente, dada a dificuldade de gerenciamento de informação e baixa aderência de pacientes. A utilização de dispositivos móveis em diferentes

In: XVII Workshop de Ferramentas e Aplicações (WFA 2018), Salvador, Brasil. Anais do XXIV Simpósio Brasileiro de Sistemas Multimídia e Web: Workshops e Pôsteres.

Porto Alegre: Sociedade Brasileira de Computação, 2018.

(C) 2018 SBC - Sociedade Brasileira de Computação.

ISBN 978-85-7669-435-9. cenários de tratamento já demonstrou ser eficiente em aumentar a aderência de participantes [10].

Intervenções personalizadas também apresentam utilidade em outros contextos, como o educacional. A utilização de dispositivos móveis no ensino à distância, designado pelo termo m-learning, e a criação de conteúdo sob demanda para diferentes perfis de alunos, tem se mostrado uma estratégia eficiente no aprendizado, promovendo maior motivação e engajamento [16].

O Método de Amostragem de Experiências (ESM) [6] é um método verificado e bem-sucedido para a coleta dados sobre as pessoas em seu ambiente natural e, apesar de proposto em papel, seu uso em dispositivos computacionais tornou-se uma alternativa viável. Já no contexto interventivo, a abordagem de Sistema Personalizado de Instrução (PSI) [8] mostrou-se eficiente em melhorar o aprendizado. Com o objetivo de criar um método que permita coletar informações e gerar conteúdo instrucional personalizado via dispositivos móveis, em trabalhos anteriores, os autores propuseram o Método de Amostragem de Experiências e Intervenção Programada (ESPIM) [14] que faz uso da Computação Ubíqua apoiada pelos métodos ESM e PSI. As ferramentas apresentadas neste trabalho são apoiadas pelo método ESPIM.

O conjunto de ferramentas busca prover a autoria de programas interventivos, que podem ser compostos por intervenções de coleta ou de conteúdos instrucionais, por parte de profissionais e especialistas de saúde e educação, assim como a reprodução de telas interativas em dispositivos móveis para a interação com os públicos alvo. Assim foram desenvolvidos um sistema Web para a autoria dos programas de intervenção personalizada que gera documentos JSON que representam os programas e um aplicativo para smartphones e tablets com sistema Android, que recebe os documentos e gera dinamicamente as telas de interação, bem como as notificações disparadas em horários definidos durante a autoria. Este trabalho objetiva detalhar as ferramentas citadas.

\section{TRABALHOS RELACIONADOS}

No contexto de mhealth, muitos aplicativos interventivos foram propostos com o intuito de coletar dados [18], disparar lembretes de medicação [5], aumentar a motivação e aderência em atividades [4], enviar instruções ou informações [9], ou uma combinação destas funcionalidade para realizar acompanhamentos em condições específicas $[1,11]$. No entanto, esses aplicativos foram implementados por profissionais de computação, seguindo requisitos de domínios específicos, sem permitir a flexibilidade de customização por profissionais de outros domínios.

Considerando aplicações computacionais que utilizam a metodologia ESM para coletar dados, Berkel et al. [3] apontam a falta 
de ferramentas que permitam a autoria de experimentos por parte de especialistas. A criação de experimentos que utilizam ESM e dispositivos móveis está bastante atrelada a necessidade de um profissional com conhecimentos de programação.

Procurando apresentar uma forma de personalização, o framework AWARE [7] permite a criação de questões ESM personalizadas, no entanto, sua programação deve ser feita em um arquivo JSON. A ExperienceSampler [17], apesar de afirmar propor uma interface de autoria simplificada, exige que o profissional execute uma série linhas de comandos e também faça suas configurações em um arquivo de texto. A plataforma PACO [2] permite que pesquisadores realizem a autoria de experimentos personalizados por meio de um Website e que usuários se registrem em experimentos em seus dispositivos móveis. Sua interface de autoria contém termos da área da Computação, principalmente relacionada à programação, e configurações complexas, o que dificulta seu entendimento para leigos. A ferramenta Ohmage [15], por sua vez, permite criar experimentos personalizados com questões específicas, porém apresenta pouca flexibilidade em termos de configuração de intervenções, não permitindo, por exemplo, a visualização e captura de mídias.

A ilumivu ${ }^{1}$ é uma plataforma paga que, apesar de apresentar um aplicativo móvel com interface convidativa, possui uma interface de autoria complexa e limitada, além de apenas permitir questões com respostas em escala ou texto. O movisensXS ${ }^{2}$ é um sistema que permite a criação de experimentos ESM que pode ser considerado um sistema que representa bem o estado-da-arte, no entanto sistema é pago e não possui código aberto, de forma que é pouco customizável ou extensível.

As ferramentas citadas apresentam limitações em termos de interação com os usuários, tanto na autoria quanto na reprodução. Este trabalho visa apresentar uma alternativa mais completa no contexto de criação de programas interventivos, que permita a criação de conteúdos textuais e multimídia, assim como a solicitação de resposta em formato multimídia.

\section{ESPIM: SISTEMAS PARA AUTORIA E REPRODUÇÃO DE INTERVENÇÕES}

O sistema ESPIM utiliza o Design Participativo como estratégia de desenvolvimento, de modo que especialistas das áreas da computação, saúde e educação estão envolvidos desde a etapa de levantamento de requisitos até as fases de avaliação e validação. $\mathrm{O}$ sistema é composto por um modelo e por duas ferramentas, sendo uma de autoria e outra de reprodução. É importante destacar que as interfaces das ferramentas aqui apresentadas contaram com o apoio de designers experientes na criação de interfaces gráficas, de forma que, além de aprimorar a experiência do usuário, o design atual apresenta padrões estéticos de um produto comercial. As próximas seções buscam detalhar a arquitetura do sistema e suas ferramentas.

\subsection{Aplicação Web para Autoria}

O objetivo da ferramenta de autoria é permitir que profissionais, especialistas das áreas de saúde e educação, elaborem programas de intervenção personalizados e interativos, que contenham texto, questões e mídias, para serem exibidos nos dispositivos móveis de

\footnotetext{
${ }^{1}$ https://ilumivu.com/

${ }^{2}$ https://xs.movisens.com/
}

participantes cadastrados. Além de criar os programas, a ferramenta permite que os profissionais visualizem os resultados das interações de seus participantes com os programas em seus dispositivos.

A ferramenta de autoria trata-se de uma Rich Internet Applicatiom (RIA), desenvolvida fazendo o extenso uso de JavaScript. A ferramenta de autoria também contém um módulo de visualização de resultados. Toda a aplicação foi desenvolvida utilizando HTML5, CSS e o framework CSS Bootstrap, seguindo princípios de Material Design ${ }^{3}$ e design responsivo. A interface de autoria utiliza o framework JavaScript Angular.js, enquanto a de visualização de resultados utiliza os frameworks JavaScript React, D3.js e plotly.js, sendo os dois últimos voltados especificamente para a visualização de dados e geração de gráficos respectivamente.

A interface de autoria permite aos especialistas criar, exportar e importar programas personalizados para coleta de dados, para disponibilização de informações ou para intervenção remota de participantes, os quais devem ser incluídos nos programas de interesse. Esses programas também podem ter observadores, tais como outros colegas profissionais, parentes ou cuidadores. Um programa é composto por um conjunto de intervenções, agrupadas em uma entidade denominada evento. Um evento contém disparos temporais que podem ser de diferentes tipos: uma notificação discreta, uma notificação longa ou um alarme. Um participante também pode iniciar a reprodução manualmente clicando no evento listado no aplicativo. Durante a configuração do evento o especialista pode bloquear ou permitir a inicialização manual pelo participante.

As intervenções são as unidades de interação com o usuário final, ou seja, representam o conteúdo exibido e requisitado aos participantes em seus dispositivos móveis. Os seguintes tipos de intervenção estão disponíveis para criação: questões abertas, questões de múltipla escolha ou escolha única, mensagens, solicitação de mídias (áudio, vídeo, imagem) e aplicação externa (em que um aplicativo externo pode ser iniciado). O enunciado das intervenções pode conter mídias do tipo imagem, áudio e vídeo, enviadas pelo especialista via interface de gravação e upload de mídias.

A interface de autoria de intervenções utiliza uma tela semelhante a um fluxograma dinâmico, que oferece uma maneira flexível e visual de conectá-las, criando fluxos sequenciais. A Figura 1 ilustra a tela para a criação das intervenções usando a interface Web de autoria do sistema.

A aplicação Web dispõe de um módulo para a visualização e análise dos dados coletados, o qual pode ser acessado por qualquer observador do programa de interesse. $\mathrm{Na}$ área de resultados, após selecionar um programa de interesse, o observador recebe uma tela que apresenta a quantidade de resultados recuperados até o momento e o número de execuções do evento por participantes. Nesta tela o observador tem a opção de baixar um arquivo do tipo Comma Separated Value (CSV), que contém todos os dados coletados referentes às respostas de todos os participantes, ou realizar uma visualização detalhada dos dados por participante. Essa última tela contém recursos gráficos para análise de resultados.

A visualização por participante disponibiliza o detalhamento de suas interações com as intervenções. Com base nos objetos retornados pelo documento JSON, oriundos das respostas aos programas, quatro representações gráficas são exibidas. Elas disponibilizam

\footnotetext{
${ }^{3}$ https://material.io/design/
} 


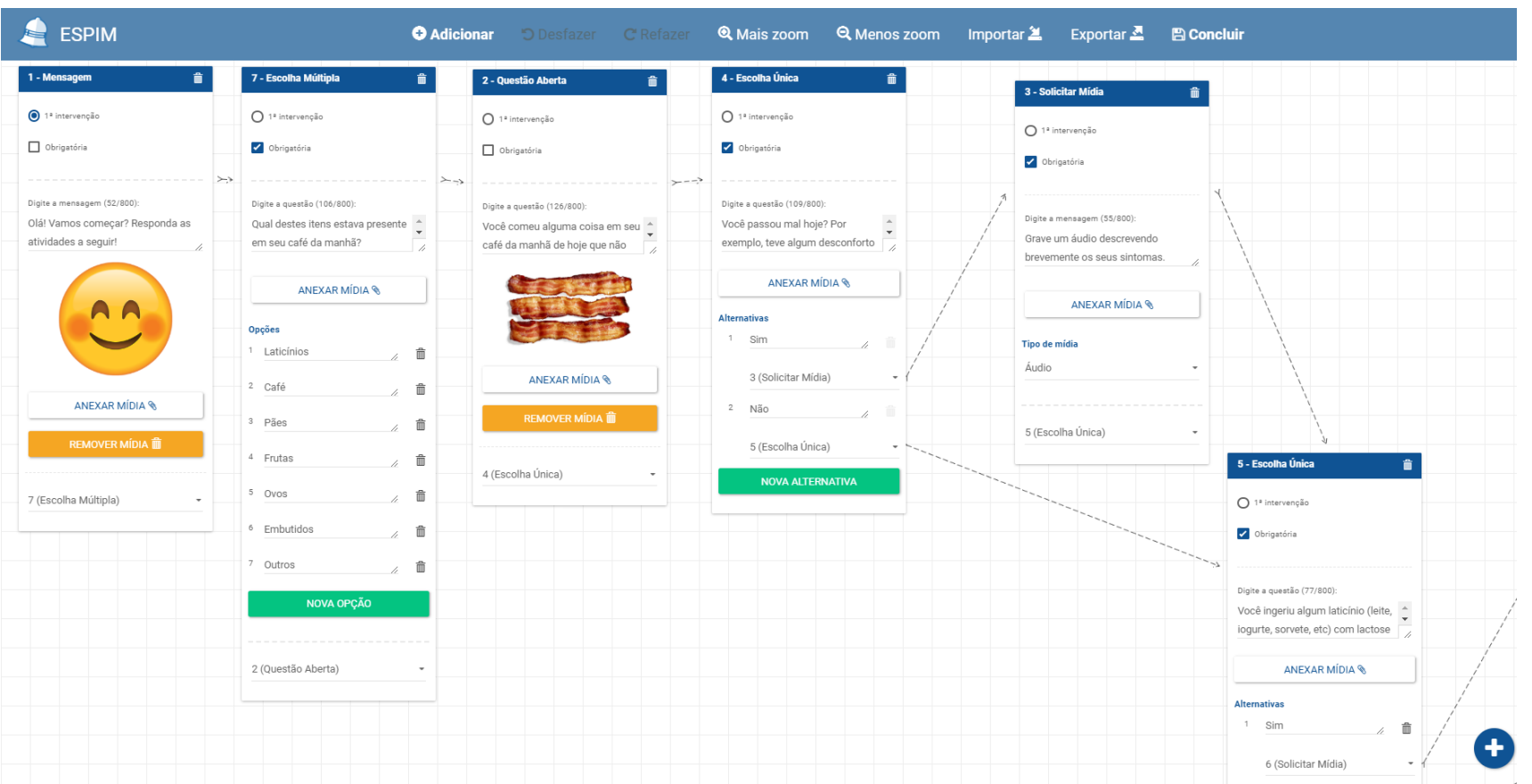

Figura 1: Exemplo da interface de autoria de intervenções.

informações tais como: em qual dia determinado participante mais respondeu às intervenções planejadas, em qual período do dia essa interação aconteceu, quais são as intervenções mais respondidas e quais intervenções os participantes levam mais tempo para concluir a interação. Além dos gráficos, a tela possui uma tabela com todos os resultados coletados. Na tabela, para cada intervenção é exibido seu tipo, horário do início da interação, horário do fim da interação, o enunciado da intervenção e o resultado enviado pelo usuário, caso aplicável.

A aplicação para autoria de programas ESPIM está disponível em: https://goo.gl/YPvwmb.

\subsection{Aplicativo Móvel para Reprodução}

O aplicativo Smart ESPIM aNd Sensor Mobile (SENSEM) é responsável por recuperar os programas ESPIM criados via aplicação Web e gerar sob demanda as telas de intervenções, de acordo com o que foi configurado durante a autoria. Em sua primeira execução o aplicativo apresenta telas de apresentação e pede para que o usuário realize o login usando a sua conta Google. Após o login, é realizada uma requisição REST para verificar se o usuário está cadastrado em algum programa e, em caso positivo, os programas são recuperados. O Web Service retorna ao aplicativo um documento JSON que contém todos os programas em que esse usuário foi cadastrado e é com base neste documento que as telas são geradas.

Como já citado, as telas são baseadas nas as seguintes possibilidades de intervenções: mensagens (as quais apresentam apenas conteúdo), questões abertas, questões de múltipla escolha com única ou múltipla seleção, captura de mídias (foto, áudio e vídeo) e inicialização de aplicações externas. Todas as telas seguem um determinado template estruturado, de forma que o texto aparece sempre no topo da tela e em seguida é apresentada a mídia opcional, caso presente. Abaixo deste conteúdo é apresentada a interação que o usuário deve realizar, que pode ser obrigatória ou não. A Figura 2 ilustra exemplos de telas do aplicativo.

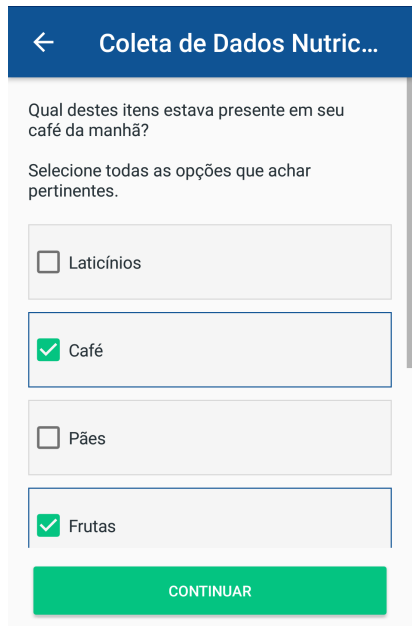

(a) Enunciado textual

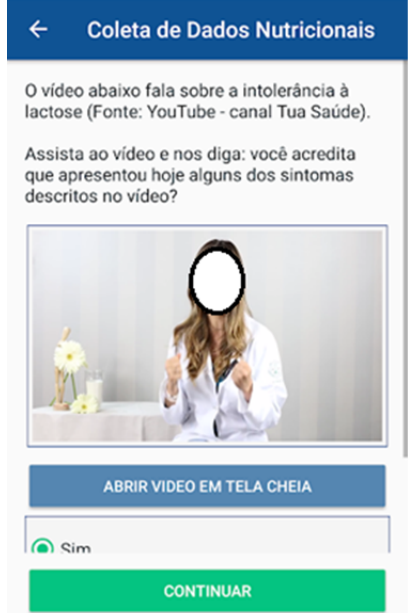

(b) Enunciado multimídia

Figura 2: Telas do aplicativo com questões de múltipla escolha e diferentes enunciados.

Intervenções que solicitam a captura de mídias são apresentadas de forma diferenciada, de acordo com a mídia solicitada para facilitar a interação com usuário. No caso de vídeo e foto é exibida uma tela 
similar que invoca a câmera para gravação (Figura 3 (a)). Já para a gravação de áudio é exibido um botão com a instrução "Segure para gravar” (Figura 3 (b)). Após a primeira captura, a mídia capturada pode ser reproduzida na própria tela e o usuário pode optar por capturar novamente caso não fique satisfeito com o resultado.

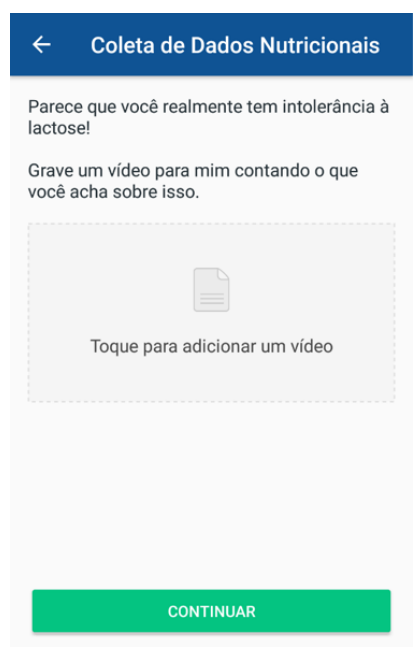

(a) Gravação de vídeo

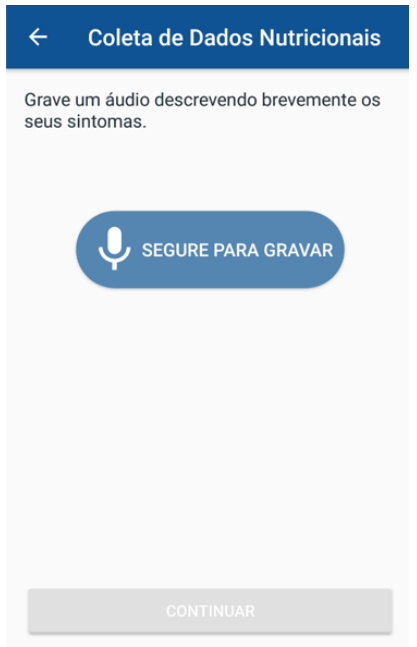

(b) Gravação de áudio
Figura 3: Telas do aplicativo para captura de mídias.

Durante a interação é verificada a obrigatoriedade das intervenções, assim como as opções que podem levar a diferentes fluxos. $\mathrm{O}$ usuário pode voltar e alterar o valor de respostas anteriores, o que pode alterar o fluxo posterior. Para isso, foi implementado um algoritmo que realiza o controle do caminho percorrido e das respostas válidas respondidas durante a interação.

Os disparos temporais, configurados no evento pela interface de autoria, apresentam três formatos: uma notificação com som padrão do sistema, uma notificação com um alerta sonoro de dez segundos e um alarme, o qual abre a aplicação na tela e toca um alerta sonoro por um minuto ou até o usuário interagir com o aplicativo.

Quando o usuário finaliza a interação com um conjunto de intervenções, os dados da interação são armazenados localmente no dispositivo e, caso haja conexão com a Internet, enviados instantaneamente para o Web Service, caso contrário, o aplicativo enviará os dados quando houver uma conexão disponível. O aplicativo também apresenta funcionalidades de limpeza automática e manual de mídias, de forma a não sobrecarregar o armazenamento dos dispositivos.

O aplicativo SENSEM para reprodução de programas ESPIM está disponível em: https://goo.gl/yDXUrp. O SENSEM é compatível com smartphones e tablets com sistema operacional Android à partir da versão KitKat (4.4) e é compilado e testado na sua versão mais recente $(8.0, \text { Oreo })^{4}$.

\footnotetext{
${ }^{4}$ https://www.android.com/versions/oreo-8-0/
}

\subsection{Arquitetura}

A arquitetura do sistema buscou criar independência entre seus componentes e dados. Desta forma, a arquitetura REST e o uso de armazenamento de dados na nuvem foram as estratégias escolhidas. Assim, toda a comunicação entre os componentes é realizada por meio da Internet, como mostra a Figura 4.

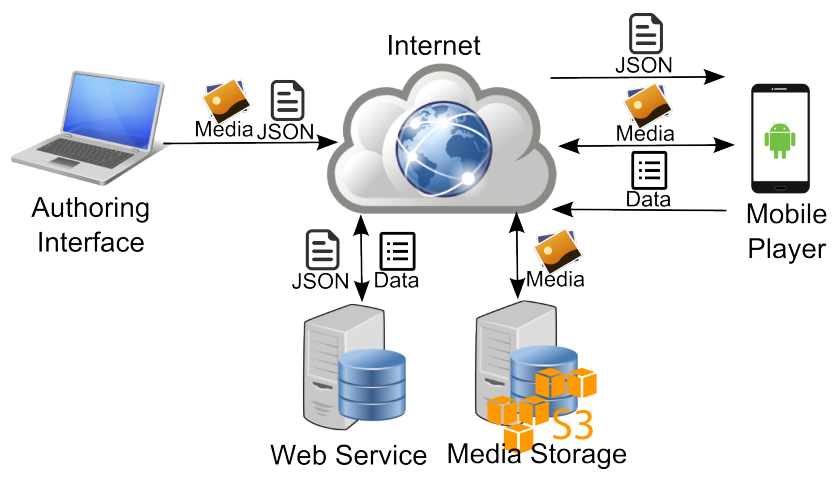

Figura 4: Arquitetura simplificada do sistema.

O modelo de dados para o método ESPIM foi concebido considerando um conjunto amplo de requisitos que foram elicitados, inicialmente, por uma revisão da literatura de artigos que abordavam ESM e ferramentas mhealth e, mais extensivamente, por entrevistas com profissionais da área da saúde e educação, as quais geraram uma grande variedade de particularidades, estas previstas no modelo de forma a permitir generalização e extensão das possibilidades de construção dos programas.

Os dados de programas interventivos, armazenados no formato do modelo proposto, são acessados por um Web Service REST, de forma que a comunicação entre as ferramentas é feita pela troca de documentos JSON. As mídias no formato de áudio, imagem e vídeo são armazenadas em um servidor independente. Neste caso, é utilizado o serviço Amazon S3 ${ }^{5}$, devido sua alta capacidade de armazenamento. Ao salvar um programa na ferramenta de autoria, o documento JSON resultante é enviado ao Web Service, enquanto as mídias são enviadas para o servidor de mídias. O documento JSON possui apenas os endereços URL das mídias armazenadas, de forma que o documento transporta apenas informação textual.

O aplicativo Android realiza requisições para recuperar documentos JSON referentes aos programas em que o usuário está cadastrado. As mídias são recuperadas por meio de suas URLs disponíveis nos documentos. O aplicativo também envia documentos JSON, que contém os resultados das interações realizadas pelos usuários, para serem armazenados pelo Web Service, e mídias capturadas para o servidor S3.

Atualmente o cadastro e login no sistema, tanto para autores quanto para reprodutores de programas, é realizado por meio da conta Google deste usuário. No caso do especialista, autor de programas, o cadastro é feito na primeira vez que acessa o sistema, utilizando a opção de Sign in with Google na primeira página da aplicação Web. Os especialistas precisam dos endereços de e-mail Google dos participantes para cadastra-los nos programas. Já no

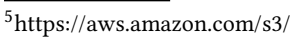


aplicativo, dada a integração da conta Google com o sistema Android, o login é realizado ao selecionar a conta desejada. Esta abordagem foi utilizada por questões de segurança, já que utiliza um sistema seguro para gerenciamento de login e senha.

\section{AVALIAÇÕES}

Durante o processo de concepção do sistema ESPIM foram realizadas avaliações em suas ferramentas com a colaboração de especialistas de diferentes áreas. Entre as avaliações realizadas estão: testes empíricos com usuários, inspeções heurísticas de usabilidade e um teste de usabilidade com usuários. Ressalta-se aqui, que este projeto foi aprovado pelo Comitê de Ética em Pesquisa com número de processo 57875016.3.0000.5390.

A interface Web de autoria está em sua 5a versão e foi submetida a uma avaliação heurística com pesquisadores da área Interação Humano-Computador e a um teste de usabilidade com usuários profissionais das áreas de terapia ocupacional e gerontologia. No momento o time concentra esforços em oferecer recursos de ajuda e documentação para a ferramenta, tais como vídeos instrucionais e uma Wiki.

O aplicativo SENSEM está em sua 3- versão e passou por duas avaliações de inspeção heurística, além de estar sendo utilizado por diferentes especialistas (gerontólogos, terapeutas ocupacionais e psicólogos) em intervenções e coletas de dados com suas populações de interesse. Entre as coletas cita-se: lembretes de atividades para pais de crianças autistas (psicologia), reforço em cursos de letramento digital para idosos (terapia ocupacional e computação), autoria de instruções para cuidadores (gerontologia) e compartilhamento de mídias para aprimorar relacionamento entre pessoas idosas (psicologia). A condução destes experimentos com usuários reais foi de extrema importância para revelar bugs de software e problemas de usabilidade, os quais foram corrigidos no decorrer de sua execução. No momento o time está trabalhando na disponibilização de disparos contextuais de eventos.

\section{CONCLUSÃO}

A versão atual do sistema representa um esforço conjunto do time de pesquisadores e desenvolvedores que compõe a equipe deste projeto, na busca constante pelo aperfeiçoamento de toda a infraestrutura. Os desenvolvedores têm realizado manutenções corretivas e evolutivas no sistema a partir de testes e do feedback coletado com as avaliações empíricas e analíticas realizadas.

O sistema ESPIM tem sido usado por terapeutas ocupacionais, gerontólogos, psicólogos e professores para a coleta de dados e intervenções com suas populações de interesse, tanto em suas práticas profissionais, quanto de pesquisa. A adesão e uso do sistema por esses e outros profissionais revelam o potencial do mesmo para atividades diversas, incluindo atividades de monitoramento, compartilhamento de mídias, informes à população, entre outros.

O objetivo do grupo de pesquisa é fornecer o sistema no formato de software livre, para que outros grupos de pesquisa e profissionais possam usar e/ou modificar o sistema para atender aos seus interesses profissionais ou de pesquisa. Os códigos das ferramentas serão distribuídos sob a licença GNU General Public License (GPLv3) ${ }^{6}$. Um

\footnotetext{
${ }^{6}$ https://www.gnu.org/licenses/gpl-3.0.html
}

tutorial mostrando a criação e exibição de um programa ESPIM está disponível em https://youtu.be/wcly811tGjQ.

\section{AGRADECIMENTOS}

O presente trabalho foi realizado com apoio da CAPES, FAPESP e CNPq.

\section{REFERENNCIAS}

[1] Ana Correia Barros, João Cevada de, Àgels Bayés, Sheila Alcaine, and Berta Mestre. 2013. User-centred Design of a Mobile Self-management Solution for Parkinson's Disease. In Proceedings of the 12th International Conference on Mobile and Ubiquitous Multimedia. ACM, Article 23, 10 pages.

[2] Kathy K Baxter, Anna Avrekh, and Bob Evans. 2015. Using experience sampling methodology to collect deep data about your users. In Proceedings of the 33rd Annual ACM Conference Extended Abstracts on Human Factors in Computing Systems. ACM, 2489-2490.

[3] Niels Van Berkel, Denzil Ferreira, and Vassilis Kostakos. 2017. The Experience Sampling Method on Mobile Devices. Comput. Surveys 50, 6 (2017), 93.

[4] Paulina Bondaronek, Elizabeth Murray, and Fiona Hamilton. 2017. The Public Health Potential of the Current Health Apps for Increasing Physical Activity. In Proceedings of the 2017 International Conference on Digital Health (DH '17). ACM, New York, NY, USA, 229-230.

[5] Nichapat Boonnuddar and Pongpisit Wuttidittachotti. 2017. Mobile Application: Patients' Adherence to Medicine In-take Schedules. In Proceedings of the International Conference on Big Data and Internet of Thing (BDIOT2017). ACM, New York, NY, USA, 237-241.

[6] M. Csikszentmihalyi and R. Larson. 1987. Validity and reliability of the Experience-Sampling Method. The fournal of Nervous and Mental Disease 175, 9 (Sept. 1987), 526-536.

[7] Denzil Ferreira, Vassilis Kostakos, and Anind K Dey. 2015. AWARE: mobile context instrumentation framework. Frontiers in ICT 2 (2015), 6.

[8] Fred S. Keller. 1968. "Good-bye, teacher...". Journal of Applied Behavior Analysis 1, 1 (1968), 79-89.

[9] Pei-Yi (Patricia) Kuo and Michael S. Horn. 2017. Daily Challenges for Sustainable Lifestyles: Design Implications from a Mobile Intervention Study. In Proceedings of the 2017 ACM International foint Conference on Pervasive and Ubiquitous Computing and Proceedings of the 2017 ACM International Symposium on Wearable Computers (UbiComp '17). ACM, New York, NY, USA, 635-641.

[10] Nicholas D. Lane, Mu Lin, Mashfiqui Mohammod, Xiaochao Yang, Hong Lu, Giuseppe Cardone, Shahid Ali, Afsaneh Doryab, Ethan Berke, Andrew T. Campbell, and Tanzeem Choudhury. 2014. BeWell: Sensing Sleep, Physical Activities and Social Interactions to Promote Wellbeing. Mobile Networks and Applications 19, 3 (June 2014), 345-359.

[11] David C. Mohr, Enid Montague, Colleen Stiles-Shields, Susan M. Kaiser, Christopher Brenner, Eric Carty-Fickes, Hannah Palac, and Jenna Duffecy. 2015. MedLink: A Mobile Intervention to Address Failure Points in the Treatment of Depression in General Medicine. In Proceedings of the 9th International Conference on Pervasive Computing Technologies for Healthcare (PervasiveHealth '15). ICST (Institute for Computer Sciences, Social-Informatics and Telecommunications Engineering), ICST, Brussels, Belgium, Belgium, 100-107.

[12] Editorial Office. 2017. mHealth is now indexed in PubMed/PMC: a tremendous step forward. mHealth 3 (March 2017).

[13] Monica E. Peek. 2017. Can mHealth Interventions Reduce Health Disparities among Vulnerable Populations? Diversity \& Equality in Health and Care 14, 2 (March 2017).

[14] Kamila R.H. Rodrigues, Caio C. Viel, Isabela Zaine, Bruna C.R. Cunha, Leonardo F. Scalco, and Maria G.C. Pimentel. 2017. Data Collection and Intervention Personalized As Interactive Multimedia Documents. In Proceedings of the 23rd Brazillian Symposium on Multimedia and the Web. ACM, New York, NY, USA, 57-60.

[15] Hongsuda Tangmunarunkit, Cheng-Kang Hsieh, Brent Longstaff, S Nolen, John Jenkins, Cameron Ketcham, Joshua Selsky, Faisal Alquaddoomi, Dony George, Jinha Kang, et al. 2015. Ohmage: A general and extensible end-to-end participatory sensing platform. ACM Transactions on Intelligent Systems and Technology 6, 3 (2015), 38.

[16] Liane MR Tarouco, MCJM Fabre, Anita RS Grando, and Mary LP Konrath. 2004. Objetos de Aprendizagem para M-learning. In Florianópolis: SUCESU-Congresso Nacional de Tecnologia da Informação e Comunicação.

[17] Sabrina Thai and Elizabeth Page-Gould. 2017. ExperienceSampler: An OpenSource Scaffold for Building Smartphone Apps for Experience Sampling. (2017).

[18] Zhi Zheng, Loren Bruns, Jr, Jiajie Li, and Richard O. Sinnott. 2017. A Mobile Application and Cloud Platform Supporting Research into Alcohol Consumption. In Proceedings of the 1st International Conference on Medical and Health Informatics 2017 (ICMHI '17). ACM, New York, NY, USA, 48-55. 